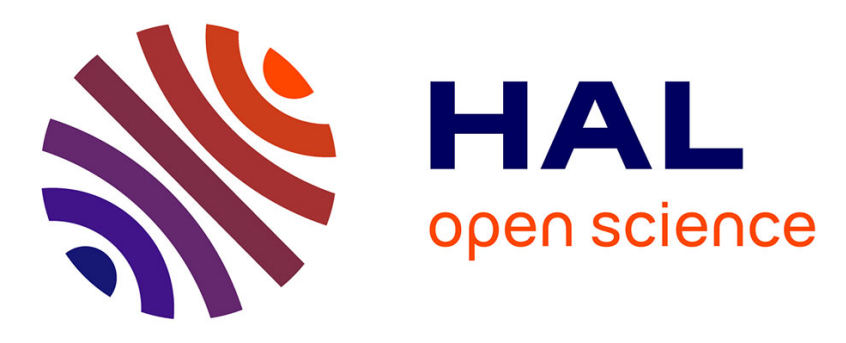

\title{
Rigid motions in the cubic grid: A discussion on topological issues
}

\author{
Nicolas Passat, Yukiko Kenmochi, Phuc Ngo, Kacper Pluta
}

\section{To cite this version:}

Nicolas Passat, Yukiko Kenmochi, Phuc Ngo, Kacper Pluta. Rigid motions in the cubic grid: A discussion on topological issues. Discrete Geometry for Computer Imagery (DGCI), 2019, Marne-laVallée, France. pp.127-140, 10.1007/978-3-030-14085-4_11. hal-01892944v2

\section{HAL Id: hal-01892944 \\ https://hal.science/hal-01892944v2}

Submitted on 9 Jan 2019

HAL is a multi-disciplinary open access archive for the deposit and dissemination of scientific research documents, whether they are published or not. The documents may come from teaching and research institutions in France or abroad, or from public or private research centers.
L'archive ouverte pluridisciplinaire HAL, est destinée au dépôt et à la diffusion de documents scientifiques de niveau recherche, publiés ou non, émanant des établissements d'enseignement et de recherche français ou étrangers, des laboratoires publics ou privés. 


\title{
Rigid Motions in the Cubic Grid: A Discussion on Topological Issues ${ }^{\star}$
}

\author{
Nicolas Passat ${ }^{1}$, Yukiko Kenmochi ${ }^{2}$, Phuc Ngo ${ }^{3}$, and Kacper Pluta ${ }^{4}$ \\ 1 Université de Reims Champagne-Ardenne, CReSTIC, France \\ 2 Université Paris-Est, LIGM, CNRS - ENPC - ESIEE Paris - UPEM, France \\ 3 Université de Lorraine, LORIA, UMR 7503, Vandoeuvre-lès-Nancy, F-54506, France \\ 4 Technion - Israel Institute of Technology, Haifa, Israel
}

\begin{abstract}
Rigid motions on 2D digital images were recently investigated with the purpose of preserving geometric and topological properties. From the application point of view, such properties are crucial in image processing tasks, for instance image registration. The known ideas behind preserving geometry and topology rely on connections between the 2D continuous and 2D digital geometries that were established via multiple notions of regularity on digital and continuous sets. We start by recalling these results; then we discuss the difficulties that arise when extending them from $\mathbb{Z}^{2}$ to $\mathbb{Z}^{3}$. On the one hand, we aim to provide a discussion on strategies that prove to be successful in $\mathbb{Z}^{2}$ and remain valid in $\mathbb{Z}^{3}$; on the other hand, we explain why certain strategies cannot be extended to the 3D framework of digitized rigid motions. We also emphasize the relationships that may exist between certain concepts initially proposed in $\mathbb{Z}^{2}$. Overall, our objective is to initiate an investigation about the most promising approaches for extending the $2 \mathrm{D}$ results to higher dimensions.
\end{abstract}

\section{Introduction}

Geometric transformations are often involved in 2D and 3D digital image processing such as image registration [26]. Among them, rigid motions, i.e. translations, rotations and their composition, are fundamental ones. When a rigid motion is applied to a digital image, we need to digitize the result in order to map back each point onto the Cartesian grid. In such a point-wise model of rigid motions in $\mathbb{Z}^{n}$, this final digitization step induces discontinuities in the transformation space. A direct consequence is the loss of geometric and topological invariance during rigid motions in $\mathbb{Z}^{n}$, as shown in Fig. 1, by contrast with rigid motions in $\mathbb{R}^{n}$ where geometry and topology are preserved.

These topological issues have been studied in $\mathbb{Z}^{2}$. In particular, a class of digital images that preserve their topological properties during rigid motions-called regular images-was identified, as well as the "regularization" process based on an upsampling strategy. This regularization approach allows to create regular images out of

\footnotetext{
* This work was funded by the French Agence Nationale de la Recherche, grant agreements ANR-15-CE40-0006 (CoMeDiC, https://lama.univ-savoie.fr/comedic) and ANR-15CE23-0009 (MAIA, http://recherche.imt-atlantique.fr/maia) ; and by the French Programme d'Investissements d'Avenir (LabEx Bézout, ANR-10-LABX-58).
} 

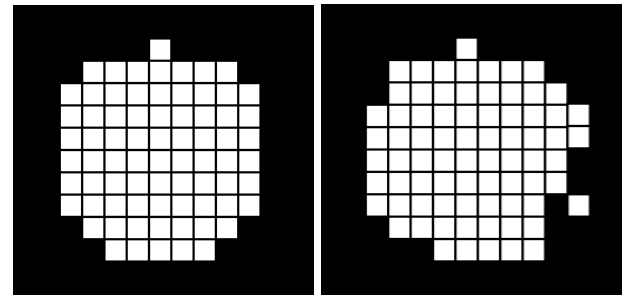

(a)

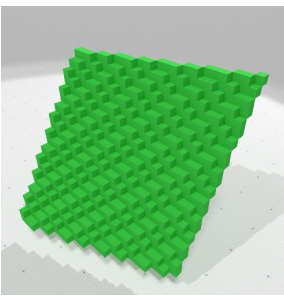

(b)

Fig. 1: Digital images (left) and their images under digitized rigid motions (right). (a) A digital disk. (b) A digital plane. These sets preserve neither topology nor geometry.

non-regular ones [18]. However, this up-sampling strategy in $\mathbb{Z}^{2}$ cannot be directly extended to $\mathbb{Z}^{3}$, leading to topological open problems in $3 \mathrm{D}$ digitized rigid motions [1]. In this paper, we investigate this topic.

In particular, we consider an alternative approach to regularity, based on quasi- $r$ regular polygons, which are used as intermediate continuous models of digital shapes for their rigid motions in $\mathbb{Z}^{2}$ [16]. This approach, which relies on a mixed dicretecontinuous paradigm (see [9] for related works), relies on three steps: polygonizing the boundary of a given digital set; applying a rigid motion on the polygon; and digitizing the transformed polygon. Topological issues may occur during the last step. In this context, the class of quasi-r-regular polygons provides guarantees on topological preservation between the polygons and their digitized analogue. It should be mentioned that quasi-r-regularity is related to the classical notions of $r$-regularity [20] and $r$-halfregularity [25] for continuous sets with smooth and polygonal boundaries, respectively. The main advantage of this approach is its possible extension to 3D [17], by contrast to the notion of regularity.

Our first contribution is a link between the two concepts of regularity and quasi- $r$ regularity, in 2D. We also show that such a link does not exist in 3D. This difference explains why a straightforward extension of image regularity to 3D does not preserve topology under the point-wise rigid motions in $\mathbb{Z}^{3}$. This fact emphasizes that quasi- $r$ regular polyhedra may be a key concept for topology-preserving 3D rigid motion on $\mathbb{Z}^{3}$. Then, a question arises: which polyhedrization method(s) can guarantee generating quasi- $r$-regular polyhedra from 3D binary images? Our first investigations show that polyhedral isosurfaces generated by the marching cubes method [12] - mostly used for $3 \mathrm{D}$ digital images - do not fulfill quasi-r-regularity requirements in $\mathbb{R}^{3}$, whereas, its $2 \mathrm{D}$ analogue, namely the marching squares method allows one to generate quasi- $r$-regular polygons in $\mathbb{R}^{2}$. 


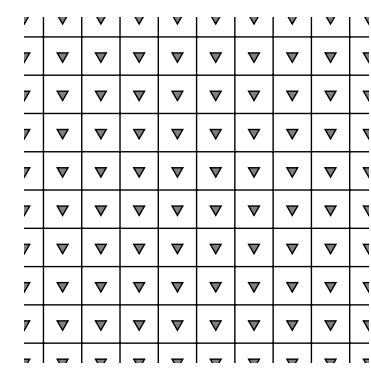

(a)

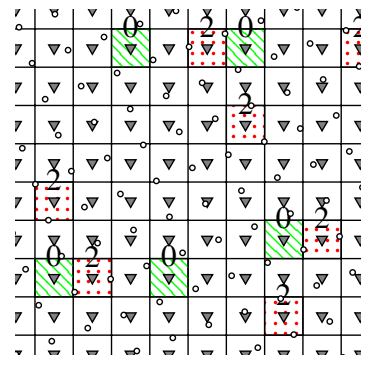

(b)

Fig. 2: (a) Points of $\mathbb{Z}^{2}$ (triangles), initially located at the center of unit squares of the Cartesian tilling of the Euclidean space. (b) After a rigid motion $\mathfrak{I}$ of these points (circles), some of the unit square cells contain no point (non-surjectivity, green cells) or two points (non-injectivity, red cells); the transformation $\mathcal{T}$ is no longer bijective.

\section{Rigid Motions on $\mathbb{Z}^{n}$}

Let us consider a bounded, closed, connected subset $X$ of the Euclidean space $\mathbb{R}^{n}, n \geq 2$. A rigid motion on $\mathbb{R}^{n}$ is defined by a mapping

$$
\begin{aligned}
\mathfrak{I}: \mathbb{R}^{n} & \rightarrow \mathbb{R}^{n} \\
\mathrm{x} & \mapsto R \mathrm{x}+\mathrm{t}
\end{aligned}
$$

where $R$ is a rotation matrix and $\mathrm{t} \in \mathbb{R}^{n}$ is a translation vector. Such bijective transformation $\mathfrak{I}$ is isometric and orientation-preserving, so that $\mathfrak{I}(X)$ has the same shape as $X$ i.e., both its geometry and topology are preserved.

If we simply apply a rigid motion $\mathfrak{I}$, such as defined in Eq. (1), to the discrete set $\mathbb{Z}^{n}$, we generally have $\mathfrak{I}\left(\mathbb{Z}^{n}\right) \nsubseteq \mathbb{Z}^{n}$. Then, in order to map back onto $\mathbb{Z}^{n}$, we need a digitization operator

$$
\mid \begin{aligned}
\mathfrak{D}: \mathbb{R}^{n} & \rightarrow \mathbb{Z}^{n} \\
\left(x_{1}, \ldots, x_{n}\right) & \mapsto\left(\left\lfloor x_{1}+\frac{1}{2}\right\rfloor, \ldots,\left\lfloor x_{n}+\frac{1}{2}\right\rfloor\right)
\end{aligned}
$$

where $\lfloor s\rfloor$ denotes the greatest integer lower than $s$. A discrete version of $\mathfrak{I}$ is then obtained by

$$
\mathcal{T}=\mathfrak{D} \circ \mathfrak{I}_{\mathbb{Z}^{n}}
$$

so that the point-wise rigid motion of a finite subset $X$ on $\mathbb{Z}^{n}$ is given by $\mathcal{T}(\mathrm{X})$. Due to the behavior of $\mathfrak{D}$ that maps $\mathbb{R}^{n}$ onto $\mathbb{Z}^{n}$, digitized rigid motions are, most of the time, non-bijective (see Fig. 2). In addition, they guarantee neither topology nor geometry preservation of X (see Fig. 1). 


\section{Regular Images and Topological Invariance under Rigid Motions}

The above problems were studied in [18] and led to the notion of regularity in $\mathbb{Z}^{2}$ defined in the frameworks of digital topology [7] and well-composed images [11] (see Sec. 3.2). Unfortunately, this notion of regularity is inadequate in $\mathbb{Z}^{3}$ (Sec. 3.3).

\subsection{Digital Topology and Well-composed Images}

Digital topology [7] provides a simple framework for handling the topology of binary images in $\mathbb{Z}^{n}$. It is shown in [14] that it is compliant with other discrete models (e.g. Khalimsky grids [5] and cubical complexes [8]) but also with continuous notions of topology [15].

Practically, digital topology relies on adjacency relations: two distinct points $p, q \in$ $\mathbb{Z}^{2}$ are $k$-adjacent if $\|\mathrm{p}-\mathrm{q}\|_{\ell} \leq 1$ with $k=2 n$ (resp. $3^{n}-1$ ) when $\ell=1$ (resp. $\infty$ ). In the case of $\mathbb{Z}^{2}$ (resp. $\mathbb{Z}^{3}$ ), we retrieve the well-known 4- and 8-adjacency (resp. 6- and 26-adjacency) relations. If two points $\mathrm{p}, \mathrm{q}$ are $k$-adjacent, we note $\mathrm{p}-_{k} \mathrm{q}$.

From the reflexive-transitive closure of the $k$-adjacency relation on a finite subset $\mathrm{X} \subset \mathbb{Z}^{n}$, we derive the $k$-connectivity relation on $\mathrm{X}$. It is an equivalence relation whose equivalence classes are called the $k$-connected components of $\mathrm{X}$. Due to paradoxes related to the discrete version of the Jordan theorem [13], some dual adjacencies are used for $\mathrm{X}$ and its complement $\overline{\mathrm{X}}=\mathbb{Z}^{n} \backslash \mathrm{X}$, namely the $(k, \bar{k})$-adjacencies [22], where $(k, \bar{k})=\left(2 n, 3^{n}-1\right)$ or $\left(3^{n}-1,2 n\right)$.

The notion of well-composedness [11] was then introduced to characterize some digital sets $\mathrm{X}$ whose structure intrinsically avoids the topological issues of the Jordan theorem in $\mathbb{Z}^{2}$ and further in higher dimensions.

Definition 1 (Well-composed sets [11]). We say that a set $\mathrm{X} \subset \mathbb{Z}^{2}$ is weakly wellcomposed if any 8-connected component of $\mathrm{X}$ is also a 4-connected component. We say that $\mathrm{X}$ is well-composed if both $\mathrm{X}$ and $\overline{\mathrm{X}}$ are weakly well-composed.

The notion of well-composedness on sets is trivially extended to binary images: an image $I: \mathbb{Z}^{2} \rightarrow\{0,1\}$, defined by the finite set $X=I^{-1}(\{1\})=\left\{p \in \mathbb{Z}^{2} \mid I(p)=1\right\}$ is well-composed when $\mathrm{X}$ is well-composed.

This definition implies that the boundary ${ }^{5}$ of $\mathrm{X}$ is a set of 1-manifolds whenever $\mathrm{X}$ is well-composed. In particular, a definition of well-composedness in $\mathbb{Z}^{n}, n \geq 3$, is based on this $(n-1)$-manifoldness characterization. This discussion is out of the scope of this paper; the interested reader is referred to $[4,10]$ for more details.

\footnotetext{
${ }^{5}$ Here, the notion of boundary is related to the continuous embedding of $\mathrm{X}$ into the Euclidean space $\mathbb{R}^{2}$. More precisely, we associate each point $p \in X$ with the closed unit square i.e., a Voronoi cell or a pixel centered in $p$. The union of these squares forms a polygon $P$ in $\mathbb{R}^{2}$, and we consider the boundary of this polygon. The way of passing from $X \subset \mathbb{Z}^{2}$ to $P \subset \mathbb{R}^{2}$ will be called "polygonization" and more extensively discussed in Sec. 4.
} 


\subsection{Topological Invariance under Rigid Motions in $\mathbb{Z}^{2}$}

Given a binary image $I$, a rigid motion $\mathcal{T}: \mathbb{Z}^{2} \rightarrow \mathbb{Z}^{2}$, and the transformed image ${ }^{6} I_{\mathcal{T}}$ obtained from $I$ and $\mathcal{T}$, a frequent question in image analysis is: "does $\mathcal{T}$ preserve the topology between $I$ and $I_{\mathcal{T}}$ ?". It is generally answered by observing the topological invariants of these images.

Among the simplest topological invariants are the Euler-Poincaré characteristics and the Betti numbers. However, these are too weak to accurately model the notion of "topology preservation" between digital images [13]. Therefore, it is necessary to consider stronger topological invariants, e.g. the (digital) fundamental group [6], the homotopy-type (considered via various notions of simple points [2,3] or simple sets [19]), or the adjacency tree [21]. The adjacency tree was considered in [18] and allowed to develop comprehensive proofs of the topology preservation properties. Indeed, in the $2 \mathrm{D}$ case, this topology preservation is equivalent to the preservation of the homotopytype [23], that is the most commonly used topological invariant in 2D image processing.

Definition 2 (Topological invariance [18]). Let I be a binary and well-composed image. We say that I is topologically invariant if any transformed image $I_{\mathcal{T}}$ has an adjacency-tree isomorphic to that of $I$.

In [18], a new notion of regularity was then introduced for 2D images.

Definition 3 (Regular image [18]). Let I $: \mathbb{Z}^{2} \rightarrow\{0,1\}$ be a non-singular ${ }^{7}$, wellcomposed image. Let $v \in\{0,1\}$. We say that I is $v$-regular if for any $\mathrm{p}, \mathrm{q} \in I^{-1}(\{v\})$, we have

$$
(\mathrm{p}-4 \mathrm{q}) \Rightarrow\left(\exists \boxplus \subseteq I^{-1}(\{v\}), \mathrm{p}, \mathrm{q} \in \boxplus\right)
$$

where $\boxplus=\{x, x+1\} \times\{y, y+1\} \subset \mathbb{Z}^{2}$. We say that $I$ is regular if it is both 0 - and 1-regular.

The regularity-which strengthens the notion of well-composedness-provides sufficient conditions for topological invariance under rigid motions.

Theorem 1 ([18]). An image I : $\mathbb{Z}^{2} \rightarrow\{0,1\}$ is topologically invariant if it is regular.

\subsection{Topological Alterations under Rigid Motions on $\mathbb{Z}^{3}$}

In $\mathbb{Z}^{2}$, Def. 3 describes a regular set $X$ (resp. its complement $\bar{X}$ ) as a cover of $2 \times 2$ squares that must locally intersect everywhere. Intuitively, the extension of this definition to $\mathbb{Z}^{3}$ would consist of considering a cover of $2 \times 2 \times 2$ cubes, that would also locally overlap everywhere. One may expect that a regular image in $\mathbb{Z}^{3}$ would also be topologically invariant. However, this is false, in general.

Indeed, for any regular image $I$ containing a connected set $X$ (composed of at least one $2 \times 2 \times 2$ cube), we can find an ad hoc transformation $\mathcal{T}$ and a point $p \in \mathbb{Z}^{3}$ such

\footnotetext{
${ }^{6}$ In practice, we consider the backward transformation model such that $\mathcal{T}=\mathfrak{D} \circ\left(\mathfrak{I}^{-1}\right)_{\mathbb{Z}^{2}}$ rather than Eq. (3), so that $\mathcal{T}$ is surjective. This means that the transformed image $I_{\mathcal{T}}=I \circ \mathfrak{D} \circ$ $\left(\mathfrak{I}^{-1}\right)_{\mathbb{Z}^{2}}=I \circ \mathcal{T}$ has no points with either no or double / conflicted values.

${ }^{7}$ An image $I$ is singular if $\exists \mathrm{p} \in \mathbb{Z}^{2}, \forall \mathrm{q} \in \mathbb{Z}^{2},\left(\mathrm{q} \frown_{4} \mathrm{p}\right) \Rightarrow(I(\mathrm{p}) \neq I(\mathrm{q}))$.
} 


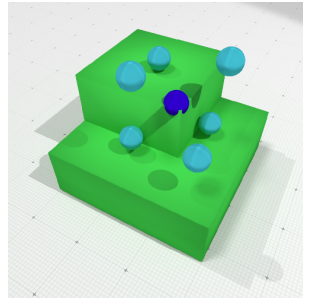

(a)

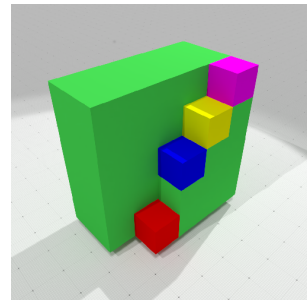

(b)

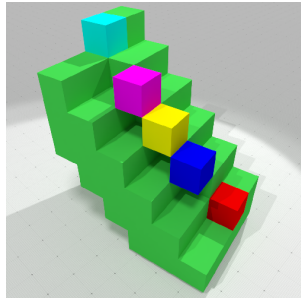

(c)

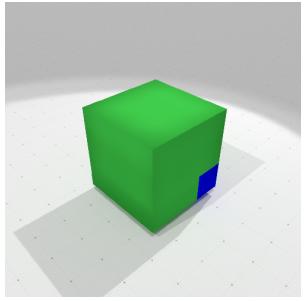

(d)

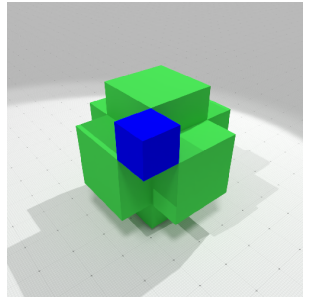

(e)

Fig. 3: (a) A sample of a regular set $\mathrm{X} \subset \mathbb{Z}^{3}$, illustrated as its voxel polyhedron $P \subset \mathbb{R}^{3}$ (in green); $X$ is 6-connected. Let us consider a rigid motion $\mathfrak{I}^{-1}$ of $\mathbb{Z}^{3}$, a part of which (transformation of a point and its 6-adjacent neighbourhood) is illustrated by blue dots. The central point (in dark blue) lies in $P$, whereas all of its 6-adjacent points (in light blue) do not. With such a transformation, $\left[\mathfrak{D} \circ\left(\mathfrak{I}^{-1}\right)\right]^{-1}(X)$ is not 6-connected anymore. $(b, c)$ A counterexample to the topology-preservation of a part of a 3D regular image under rigid motion: (b) a part of a regular set, which is 6-connected; (c) the transformed set, obtained after applying a rigid motion, which is no longer 6-connected; see, e.g. the blue voxel, which has all of its 6-adjacent points in the background. (d,e) Another counterexample to the topology-preservation of a 3D regular image under rigid motion: (d) a regular set, which is 6-connected; (e) the transformed set, which is no longer 6connected (see the blue voxel).

that $I_{\mathcal{T}}(p)=1$ whereas for any $q \neg_{6} p$ we have $I_{\mathcal{T}}(p)=0$. An example of such a case is illustrated in Fig. 3 (a). This provides us with counterexamples to the putative extension of Th. 1 to the 3D case. For an illustration, we refer the reader to Fig. 3 (b, c).

This implies that the notion of regularity reaches its limit of validity ${ }^{8}$ in $\mathbb{Z}^{2}$. Alternative approaches are then required to handle the case of topology preservation under rigid motions in higher dimensions.

\footnotetext{
${ }^{8}$ Beyond the limitations of regularity, the notion of topological invariance of Def. 2 is also insufficient in $\mathbb{Z}^{3}$. Indeed, the adjacency tree cannot model topological patterns that appear in $\mathbb{Z}^{3}$, such as the tunnels: e.g. a sphere and a torus have isomorphic adjacency trees. Considering stronger topological invariants, e.g. homotopy type or fundamental group, becomes mandatory.
} 


\section{Quasi-regular Polytopes and their Digitization}

As discussed previously, the topological properties of digital sets in $\mathbb{Z}^{n}$ may be altered by rigid motions. This is due, in particular, to the process (Eq. (3)) that aims to map back the transformed result from $\mathbb{R}^{n}$ to $\mathbb{Z}^{n}$. In practice, this issue is the same as the problem of digitization encountered for defining the digital analogue of a continuous set.

Recently, the notion of quasi-r-regularity [16] was introduced together with an algorithmic scheme in order to perform rigid motions on digital sets in $\mathbb{Z}^{2}$. The scheme relies on the use of an intermediate modeling of a $2 \mathrm{D}$ digital set as a piecewise affine subset of $\mathbb{R}^{2}$, namely a polygon. This polygon is transformed by the rigid motion, and a result in $\mathbb{Z}^{2}$ is then retrieved by a final digitization of the transformed polygon. The polygon in $\mathbb{R}^{2}$ and its digitized analogue in $\mathbb{Z}^{2}$ have the same topology if the polygon is quasi- $r$-regular.

Then, the use of an intermediate continuous model allows one to avoid the alterations induced by the standard pointwise definition of rigid motions that led to the difficulties identified in the case of regularity (see Sec. 3.2).

We recall the definitions of quasi- $r$-regularity that was initially defined in $\mathbb{R}^{2}[16]$ and then extended to $\mathbb{R}^{3}$ [17]. These definitions and associated results are developed in the case of simply connected (i.e. connected, without tunnels / holes) digital sets.

Definition 4 (Quasi- $r$-regularity [16,17]). Let $X \subset \mathbb{R}^{n}(n=2,3)$ be a bounded, simply connected set. We say that $X$ is quasi-r-regular with margin $r^{\prime}-r$ (with $r^{\prime} \geq r>0$ ) if it satisfies the following four properties:

- $X \ominus B_{r}$ is non-empty and connected,

$-\bar{X} \ominus B_{r}$ is connected,

- $X \subseteq X \ominus B_{r} \oplus B_{r^{\prime}}$

$-\bar{X} \subseteq \bar{X} \ominus B_{r} \oplus B_{r^{\prime}}$

with $\oplus, \ominus$ the standard dilation and erosion operators and $B_{r}, B_{r}^{\prime} \subset \mathbb{R}^{n}$ the closed balls of radius $r$ and $r^{\prime}$, respectively.

The Gauss digitization of a quasi- $r$-regular set $X \subset \mathbb{R}^{2}$ (namely $X \cap \mathbb{Z}^{2}$ ) is a wellcomposed set that remains simply connected, thus preserving its topological properties from $\mathbb{R}^{2}$ to $\mathbb{Z}^{2}$.

Proposition 1 ([16]). If $X \subset \mathbb{R}^{2}$ is quasi-1-regular with margin $\sqrt{2}-1$, then $X=X \cap \mathbb{Z}^{2}$ and $\overline{\mathrm{X}}=\overline{\mathrm{X}} \cap \mathbb{Z}^{2}$ are both 4 -connected. In particular, $\mathrm{X} \subset \mathbb{Z}^{2}$ is well-composed.

In [17], a similar result ${ }^{9}$ was obtained for convex quasi-r-regular sets of $\mathbb{R}^{3}$. This result is extended hereafter to any quasi- $r$-regular sets of $\mathbb{R}^{3}$.

Proposition 2 (Extended from [17]). If $X \subset \mathbb{R}^{3}$ is quasi-1-regular with margin $\frac{2}{\sqrt{3}}-1$, then $\mathrm{X}=X \cap \mathbb{Z}^{3}$ and $\overline{\mathrm{X}}=\bar{X} \cap \mathbb{Z}^{3}$ are both 6-connected.

\footnotetext{
${ }^{9}$ Erratum: In [17], the proposed proof contains an error. The 6-connectedness of $\left(X \circ B_{1}\right) \cap \mathbb{Z}^{3}$ is claimed by using [24, Th. 16] with an invalid value. We correct this error in the proposed proof of Proposition 2, that does no longer rely on [24, Th. 16].
} 
Proof. We only prove the 6-connectedness of $\mathrm{X}$; the same reasoning holds for $\bar{X}$. Let us first prove that $\left(X \circ B_{1}\right) \cap \mathbb{Z}^{3}$ is 6-connected. Let $p$ and $q$ be two distinct points of $\left(X \circ B_{1}\right) \cap \mathbb{Z}^{3}$. Let $B_{1}^{\mathrm{p}}$ and $B_{1}^{\mathrm{q}}$ be two balls of radius 1 , included in $X \circ B_{1}$ and such that $\mathrm{p} \in B_{1}^{\mathrm{p}}$ and $\mathrm{q} \in B_{1}^{\mathrm{q}}$ (such balls exist, from the definition of opening). Let $b_{\mathrm{p}}$ and $b_{\mathrm{q}}$ be the centers of $B_{1}^{\mathrm{p}}$ and $B_{1}^{\mathrm{q}}$, respectively. We have $b_{\mathrm{p}}, b_{\mathrm{q}} \in X \ominus B_{1}$, from the definition of erosion. Since $X \ominus B_{1}$ is connected in $\mathbb{R}^{3}$, there exists a continuous path $\Pi$ from $b_{\mathrm{p}}$ to $b_{\mathrm{q}}$ in $X \ominus B_{1}$. Note that for any ball $B_{1}$, we always have $B_{1} \cap \mathbb{Z}^{3}$ non-empty and 6-connected; in particular it contains at least two points of $\mathbb{Z}^{3}$. For a value $\varepsilon>0$ small enough, two balls $B_{1}$ and $B_{1}^{\prime}$ with centres distant of $\varepsilon$ are such that $B_{1} \cap B_{1}^{\prime} \cap \mathbb{Z}^{3} \neq \emptyset$. As a consequence, the union $\bigcup_{b \in \Pi} B_{1}(b) \cap \mathbb{Z}^{3}$ (with $B_{1}(b)$ the ball of center $b$ ) is a 6connected set of $\mathbb{Z}^{3}$, and $\mathrm{p}, \mathrm{q}$ are then connected in $\left(X \circ B_{1}\right) \cap \mathbb{Z}^{3}$. Our purpose is then to prove that any integer point $\mathrm{p}$ in $X \backslash\left(X \circ B_{1}\right)$ is 6-adjacent to a point of $\left(X \circ B_{1}\right) \cap \mathbb{Z}^{3}$. Let $\mathrm{p} \in X \backslash\left(X \circ B_{1}\right)$ be such point. From Def. 4, we have $\mathrm{p} \in X \subseteq X \ominus B_{1} \oplus B_{\frac{2}{\sqrt{3}}}$. Then, from the definition of dilation, there exists $b \in X \ominus B_{1}$ such that $b$ is the center of a ball $B_{\frac{2}{\sqrt{3}}}(b)$ of radius $\frac{2}{\sqrt{3}}$, and $\mathrm{p}$ is a point within this ball. The distance between $b$ and $\mathrm{p}$ is lower than $\frac{2}{\sqrt{3}}$. As $b$ is a point of $X \ominus B_{1}$, it is also the center of a ball $B_{1}(b)$ of radius 1 included in $X \circ B_{1}$. From the definition of adjacency, any point q being 6-adjacent to $\mathrm{p}$ belongs to the sphere $S_{1}(\mathrm{p})$ of radius 1 and center $\mathrm{p}$. Let us consider the intersection $D$ between $S_{1}(\mathrm{p})$ and $B_{1}(b)$. This set $D$ is a spherical dome, namely a part of the sphere $S_{1}(\mathrm{p})$ with a circular boundary $C$. This set $C$ also corresponds to the intersection of $S_{1}(\mathrm{p})$ and the 2D plane orthogonal to the line $(b \mathrm{p})$ and intersecting the segment $[b \mathrm{p}]$ at an equal distance lower than $\frac{1}{\sqrt{3}}$ from both $b$ and $\mathrm{p}$. Then, the radius of this circle $C$ is greater than $\sqrt{1^{2}-\left(\frac{1}{\sqrt{3}}\right)^{2}}=\frac{\sqrt{6}}{3}$. In particular, $C$ encompasses an equilateral triangle of edge length $\sqrt{2}$. As a consequence, the spherical dome $D$ of $S_{1}(\mathrm{p})$ bounded by this circle always contains at least one point $q-6 \mathrm{p}$. As such point $\mathrm{q}$ lies in $\left(X \circ B_{1}\right) \cap \mathbb{Z}^{3}$, it follows that $\mathrm{X}$ is 6-connected.

Remark 1. The value $r^{\prime}$ (see Def. 4), required to define the margin $r^{\prime}-1$ for quasi-1regular sets, is $\sqrt{2}=\frac{2}{\sqrt{2}}$ in $\mathbb{Z}^{2}$ and $\frac{2}{\sqrt{3}}$ in $\mathbb{Z}^{3}$. The above proof allows us to understand that in $\mathbb{Z}^{n}$, the required value $r^{\prime}$ is $\frac{2}{\sqrt{n}}$. Indeed, the crucial part of the proof is to ensure that a point $p$ of $X \backslash\left(X \circ B_{1}\right) \cap \mathbb{Z}^{n}$ remains $2 n$-adjacent to points of $\left(X \circ B_{1}\right) \cap \mathbb{Z}^{n}$. To this end, let us consider a $(n-1)$-simplex of $\mathbb{R}^{n}$, whose vertices are spatially organized as the $n$ points induced by the orthonormal basis of $\mathbb{R}^{n}$. This $(n-1)$-simplex must be encompassed by the $(n-2)$-sphere $C$ that is the intersection between the $(n-1)$-sphere $S_{1}(\mathrm{p})$ of center $\mathrm{p}$ and radius 1 and the hyperplane orthogonal to the segment [bp] and equidistant to $\mathrm{p}$ and a point $b \in X \ominus B_{1}$ defined as the centre of a ball $B_{\frac{2}{\sqrt{n}}}(b)$ that contains $\mathrm{p}$. Note that $\mathrm{p}$ is, by construction, at a distance $r^{\prime}$ from $b$. The distance between the barycentre of this $(n-1)$-simplex and its vertices, i.e. the radius of the $(n-1)$-sphere $C$ is $\sqrt{1-\frac{1}{n}}$. Since each point of $C$ is at a distance 1 of $p$ while the center of $C$ is on the segment $[b \mathrm{p}]$, at a distance $\frac{r^{\prime}}{2}$ from $\mathrm{p}$, it follows that the distance between $\mathrm{p}$ and $b$, namely $r^{\prime}$, is $\frac{2}{\sqrt{n}}$.

The following property is a direct corollary of this remark, and provides a dimensional limit of validity for the notion of quasi-1-regularity. 
Property 1. In $\mathbb{R}^{4}$, the value $r^{\prime}$ required for quasi-1-regularity is $\frac{2}{\sqrt{4}}=1$. Then, the margin $r^{\prime}-r$ is equal to 0 , in other words, no margin is permitted. The notion of quasi$r$-regularity then becomes similar to that of $r$-regularity [20]. In particular, only smooth sets of $\mathbb{R}^{4}$ can be quasi-1-regular.

\section{Links between Regularity and Quasi-r-regularity: the Cubic Polygonal Model}

We now investigate the links between the notions of regularity [18] (Sec. 3) and quasi$r$-regularity $[16,17]$ (Sec. 4). Note that we still focus on simply connected sets.

\section{$5.12 D$ Case}

The paradigm of quasi- $r$-regularity for rigid motion of digital sets $X \subset \mathbb{Z}^{2}$ acts in three steps. First, a polygon $P \subset \mathbb{R}^{2}$ is defined as a continuous representation of $\mathrm{X}$. Note that there exist various ways of carrying out polygonization. The main constraint is the coherence between the polygon $P$ and its digital analogue $X$. In particular, it is important to satisfy $P \cap \mathbb{Z}^{2}=\mathrm{X}$. Second, the polygon $P$ is transformed by $\mathfrak{I}$ (Eq. (1)). In other words, we build a new polygon $P_{\mathfrak{I}}=\mathfrak{I}(P)=\{\mathfrak{I}(x) \mid x \in P\}$. Third, the transformed polygon $P_{\mathfrak{I}} \subset \mathbb{R}^{2}$ is digitized to map the result back onto $\mathbb{Z}^{2}$. To this end, we use the Gauss digitization model, i.e. we set $\mathrm{X}_{\mathcal{T}}=P_{\mathfrak{I}} \cap \mathbb{Z}^{2}=\mathfrak{I}(P) \cap \mathbb{Z}^{2}$.

When considering the notion of regularity, at the first sight, the paradigm for rigid motion of digital sets $X \subset \mathbb{Z}^{2}$ may appear as a different one. It is however the same. In order to compute the transformed object $X_{\mathcal{T}}$ from $X$, we do not use the forward transformation model, but the backward one. More precisely, we define $X_{\mathcal{T}}$ as $[\mathfrak{D}$ 。 $\left.\left(\mathfrak{I}^{-1}\right)\right]^{-1}(\mathrm{X})$, that is $\mathrm{X}_{\mathcal{T}}=\left\{x \in \mathbb{Z}^{2} \mid \mathfrak{D} \circ\left(\mathfrak{I}^{-1}\right)(x) \in \mathrm{X}\right\}$. But this formula is equivalent ${ }^{10}$ to $\mathrm{X}_{\mathcal{T}}=\mathfrak{I}\left(P_{\square}(\mathrm{X})\right) \cap \mathbb{Z}^{2}$ where $P_{\square}(\mathrm{X}) \subset \mathbb{R}^{2}$ is the polygon defined as $P_{\square}(\mathrm{X})=\mathrm{X} \oplus \square$, with $\square \subset \mathbb{R}^{2}$ the closed, unit square centered on $(0,0)$. In other words, we implicitly apply the three-step polygonization-based algorithm involved in the context of quasi$r$-regularity, with a specific kind of polygonization, called cubic polygonization. This polygonization associates a digital set $X \subset \mathbb{Z}^{2}$ with its set of pixels, i.e. Voronoi cells in $\mathbb{R}^{2}$. In particular, it is plain that such polygonization satisfies $P_{\square}(\mathrm{X}) \cap \mathbb{Z}^{2}=\mathrm{X}$.

The question which now arises is to determine whether a regular digital set $\mathrm{X}$ leads to a quasi-1-regular polygon $P_{\square}(\mathrm{X})$. The answer is negative; this emphasizes the fact that quasi-1-regularity is a sufficient, yet non-necessary condition for topology preservation.

Property 2. The regularity of a simply connected set $X \subset \mathbb{Z}^{2}$ does not imply the quasi1-regularity of $P_{\square}(\mathrm{X})$.

To prove this property, it is sufficient to exhibit a counterexample. A simple one is the object $X \subset \mathbb{Z}^{2}$ defined as the union of two $2 \times 2$ patterns $\boxplus$ intersecting in exactly one point; for instance, $X=\{(0,0),(1,0),(0,1),(1,1),(2,1),(1,2),(2,2)\}$. This set is

\footnotetext{
10 This equivalence, presented here in the $2 \mathrm{D}$ case, holds for any dimension $n \geq 2$, with $\square$ being the unit $n$-cube.
} 
obviously regular, but the associated polygon $P_{\square}(\mathrm{X})$ is not quasi-1-regular. Indeed, we have $P_{\square}(\mathrm{X}) \ominus B_{1}=\left\{\left(\frac{1}{2}, \frac{1}{2}\right),\left(\frac{3}{2}, \frac{3}{2}\right)\right\}$, which is composed of two points in $\mathbb{R}^{2}$ and is nonconnected.

In [18] the strategy for building a regular set from a well-composed set $\mathrm{X}$ was to up-sample $\mathrm{X}$, i.e. to define a new set $\mathrm{X}_{2} \in \mathbb{Z}^{2}$ such that $(x, y) \in \mathrm{X} \Leftrightarrow(2 x, 2 y)+\{0,1\} \times$ $\{0,1\} \subseteq X_{2}$. By applying the same strategy on a regular set $X$, we can build an upsampled set $X_{2} \subset \mathbb{Z}^{2}$ which is still regular, but also quasi-1-regular. In other words, with the cubic polygonization model, regularity implies quasi-r-regularity-up to an up-sampling realized by doubling the resolution of the Cartesian grid.

Proposition 3. If a simply connected set $\mathrm{X} \subset \mathbb{Z}^{2}$ is regular, then $\mathrm{X}_{2} \subset \mathbb{Z}^{2}$ is regular and $P_{\square}\left(\mathrm{X}_{2}\right) \subset \mathbb{R}^{2}$ is quasi-1-regular with margin $\sqrt{2}-1$.

Proof. The regularity of $\mathrm{X}_{2}$ is obvious. We show the non-vacuity and connectedness of $P_{\square}\left(\mathrm{X}_{2}\right) \ominus B_{1}$ and the fact that $P_{\square}\left(\mathrm{X}_{2}\right) \subseteq P_{\square}\left(\mathrm{X}_{2}\right) \ominus B_{1} \oplus B_{\sqrt{2}}$; the same reasoning holds for $\overline{P_{\square}\left(\mathrm{X}_{2}\right)}$. Since $\mathrm{X}$ is regular, it is defined as $\mathrm{X}=S \oplus\{0,1\}^{2}$ where $S=\{(x, y) \in$ $\left.\mathrm{X} \mid(x, y)+\{0,1\}^{2} \subset \mathrm{X}\right\}$. By definition, we have $\mathrm{X}_{2}=\bigcup_{(x, y) \in S}(2 x, 2 y)+\{0,1,2,3\}^{2}$. Let $(x, y) \in S$. We have $\left((2 x, 2 y)+\{0,1,2,3\}^{2}\right) \oplus \square=\left[2 x-\frac{1}{2}, 2 x+\frac{7}{2}\right] \times\left[2 y-\frac{1}{2}, 2 y+\frac{7}{2}\right]$, and then $\left((2 x, 2 y)+\{0,1,2,3\}^{2}\right) \oplus \square \ominus B_{1}=\left[2 x+\frac{1}{2}, 2 x+\frac{5}{2}\right] \times\left[2 y+\frac{1}{2}, 2 y+\frac{5}{2}\right]$. Then we have $P_{\square}\left(\mathrm{X}_{2}\right)=\bigcup_{(x, y) \in S}(2 x, 2 y)+\{0,1,2,3\}^{2} \oplus \square=\bigcup_{(x, y) \in S}\left[2 x-\frac{1}{2}, 2 x+\frac{7}{2}\right] \times\left[2 y-\frac{1}{2}, 2 y+\frac{7}{2}\right]$ on the one hand, and $P_{\square}\left(\mathrm{X}_{2}\right) \ominus B_{1}=\mathrm{X}_{2} \oplus \square \ominus B_{1} \supseteq \bigcup_{(x, y) \in S}\left[2 x+\frac{1}{2}, 2 x+\frac{5}{2}\right] \times\left[2 y+\frac{1}{2}, 2 y+\frac{5}{2}\right]$, on the other hand. Due to the specific square structure of $\mathrm{X}_{2}$ and its regularity, we have $P_{\square}\left(\mathrm{X}_{2}\right) \ominus \square_{2}=\bigcup_{(x, y) \in S}\left[2 x-\frac{1}{2}, 2 x+\frac{7}{2}\right] \times\left[2 y-\frac{1}{2}, 2 y+\frac{7}{2}\right] \ominus \square_{2}=\bigcup_{(x, y) \in S}\left[2 x+\frac{1}{2}, 2 x+\frac{5}{2}\right] \times$ $\left[2 y+\frac{1}{2}, 2 y+\frac{5}{2}\right]$, where $\square_{2}$ is the square of edge size 2 centered on $(0,0)$. In particular, we have $P_{\square}\left(\mathrm{X}_{2}\right) \ominus \square_{2} \subseteq P_{\square}\left(\mathrm{X}_{2}\right) \ominus B_{1}$, and we note $R=\left(P_{\square}\left(\mathrm{X}_{2}\right) \ominus B_{1}\right) \backslash\left(P_{\square}\left(\mathrm{X}_{2}\right) \ominus \square_{2}\right)$ the residue between both. The non-vacuity of $P_{\square}\left(\mathrm{X}_{2}\right) \ominus B_{1}$ directly follows from the non-vacuity of $P_{\square}\left(\mathrm{X}_{2}\right) \ominus \square_{2}$. Up to a translation of $\left(-\frac{1}{2},-\frac{1}{2}\right)$ and a scaling of factor $\frac{1}{2}$, the set $P_{\square}\left(\mathrm{X}_{2}\right) \ominus \square_{2}$ is equal to $\bigcup_{(x, y) \in S}[x, x+1] \times[y, y+1]$. The existence of a continuous path between two points of $\bigcup_{(x, y) \in S}[x, x+1] \times[y, y+1]$ is equivalent to the existence of a 4-path between two points of $\bigcup_{(x, y) \in S}\{x, x+1\} \times\{y, y+1\}=\bigcup_{(x, y) \in S}(x, y)+\{0,1\}^{2}=$ $X$. Since $X$ is 4-connected in $\mathbb{Z}^{2}$, it follows that $P_{\square}\left(X_{2}\right) \ominus \square_{2}$ is connected in $\mathbb{R}^{2}$. The residue $R$ is composed of connected components of $\mathbb{R}^{2}$ (namely "triangular" shapes formed by two edges of length 1 adjacent to the border of $P_{\square}\left(\mathrm{X}_{2}\right) \ominus \square_{2}$ and a third concave, edge defined as a the quadrant of a circle of radius 1 ); the connectedness of $P_{\square}\left(\mathrm{X}_{2}\right) \ominus B_{1}=\left(P_{\square}\left(\mathrm{X}_{2}\right) \ominus \square_{2}\right) \cup R$ then follows from that of $P_{\square}\left(\mathrm{X}_{2}\right) \ominus \square_{2}$. We have $P_{\square}\left(\mathrm{X}_{2}\right)=P_{\square}\left(\mathrm{X}_{2}\right) \ominus \square_{2} \oplus \square_{2}$. Since $B_{1} \subset \square_{2} \subset B_{\sqrt{2}}$, the decreasingness of erosion and increasingness of dilation lead to $P_{\square}\left(\mathrm{X}_{2}\right) \subseteq P_{\square}\left(\mathrm{X}_{2}\right) \ominus B_{1} \oplus B_{\sqrt{2}}$.

\subsection{D Case}

As already observed in Sec. 3.3, the extension of the notion of regularity to $\mathbb{Z}^{3}$ leads to 3D regular sets that may not be topologically invariant (see Fig. 3 for examples). Actually, we have an even stronger result, regularity in 3D never leads to quasi- $r$-regularity.

Property 3. Let $\mathrm{X} \subset \mathbb{Z}^{3}$ be a simply connected, regular set. Let $P_{\square}(\mathrm{X})=\mathrm{X} \oplus \square$, with $\square \subset \mathbb{R}^{3}$ the closed, unit cube centered at $(0,0,0)$. Then $P_{\square}(\mathrm{X})$ is never quasi-1-regular with margin $\frac{2}{\sqrt{3}}-1$. 
To prove this property, it is sufficient to observe that for any salient vertex $v$ of $P_{\square}(\mathrm{X})$ (such vertex exists, as $\mathrm{X}$ is finite and $P_{\square}(\mathrm{X})$ is then bounded), the distance between $v$ and $P_{\square}(\mathrm{X}) \ominus B_{1}$ is $\sqrt{3}>\frac{2}{\sqrt{3}}$. Then, $v$ does not belong to $P_{\square}(\mathrm{X}) \ominus B_{1} \oplus B_{\frac{2}{\sqrt{3}}}$.

In particular, the up-sampling strategy proposed in the $2 \mathrm{D}$ case is useless in 3D. Indeed, in 2D this up-sampling allowed us to tackle connectedness issues in $P_{\square}(\mathrm{X}) \ominus B_{1}$, whereas in 3D connectedness issues occur in the complement part $P_{\square}(\mathrm{X}) \backslash\left(P_{\square}(\mathrm{X}) \ominus B_{1}\right)$, and the size of this residue is not impacted by increasing the resolution of the Cartesian grid.

\section{Links between Regularity and Quasi-r-regularity: the Marching Squares / Cubes Polygonal Model}

\subsection{D Case}

The above cubic polygonization is the model implicitly considered when applying a pointwise rigid motion with the backward transformation model, and with the nearest neighbour digitization operator $\mathfrak{D}$ (Eq. (2)). This trivial polygonization is directly mapped on the pixel structure of the image, leading to a poor modeling of the shape of the underlying continuous set.

There exist numerous ways of polygonizing a digital set. Here, we investigate the marching squares (MS) model, which is probably the simplest polygonization, except from the cubic one. In our case, the considered images are binary and regular. Then, the MS polygonization is the same as the cubic polygonization, except in the $2 \times 2$ configurations $(x, y)+\{0,1\}^{2}$ where one point (for instance $(x, y)$ ) belongs to $\mathrm{X}$ (resp. $\overline{\mathrm{X}}$ ) while the other three belong to $\overline{\mathrm{X}}$ (resp. $\mathrm{X}$ ). In that case, the edge of the MS polygon, noted $P_{\diamond}(\mathrm{X})$, associated to $\mathrm{X}$ is a segment between the points $\left(x, y+\frac{1}{2}\right),\left(x+\frac{1}{2}, y\right)$ (whereas the cubic polygon $P_{\square}(\mathrm{X})$ would locally have edges / segments between the points $\left(x, y+\frac{1}{2}\right),\left(x+\frac{1}{2}, y+\frac{1}{2}\right)$ and $\left.\left(x+\frac{1}{2}, y+\frac{1}{2}\right),\left(x+\frac{1}{2}, y\right)\right)$.

In the case of a regular object $\mathrm{X} \subset \mathbb{Z}^{2}$, the MS polygonization can be formalized as follows. We have $\mathrm{X}=S \oplus\{0,1\}^{2}$, where $S=\left\{(x, y) \in \mathrm{X} \mid(x, y)+\{0,1\}^{2} \subset \mathrm{X}\right\}$. By setting $S^{\prime}=\left(\frac{1}{2}, \frac{1}{2}\right)+S$, this rewrites as $\mathrm{X}=\bigcup_{(x, y) \in S^{\prime}}(x, y)+\left\{-\frac{1}{2}, \frac{1}{2}\right\}^{2}$. In other words, $S^{\prime}$ is the set of barycenters of the $2 \times 2$ square subsets of points forming $\mathrm{X}$. Let $C$ be the octagon centered on $(0,0)$, formed by the two edges $\left[\left(-\frac{1}{2}, 1\right),\left(\frac{1}{2}, 1\right)\right]$ and $\left[\left(\frac{1}{2}, 1\right),\left(1, \frac{1}{2}\right)\right]$, and the other six edges obtained by rotation of center $(0,0)$ and angles $k . \pi / 2, k=1,2,3$, of these two edges. (Note that the distance between $(0,0)$ and the four edges induced by $\left[\left(-\frac{1}{2}, 1\right),\left(\frac{1}{2}, 1\right)\right]$ (resp. $\left.\left[\left(\frac{1}{2}, 1\right),\left(1, \frac{1}{2}\right)\right]\right)$ is 1 (resp. $\left.\frac{3}{2 \sqrt{2}}>1\right)$ ). Let $G=\bigcup\left\{[p, q] \subset \mathbb{R}^{2} \mid p, q \in S^{\prime} \wedge 0<\|p-q\|_{2} \leq \sqrt{2}\right\}$. In other words, $G$ is the set of the continuous straight segments linking the points of $S^{\prime}$ that are either 4- or 8-adjacent in the grid $\mathbb{Z}^{2}+\left(\frac{1}{2}, \frac{1}{2}\right)$.

Property 4. Let $\mathrm{X}$ be a simply connected set. If $\mathrm{X}$ is regular, then $P_{\diamond}(\mathrm{X})=G \oplus C$

Despite its simplicity, this MS polygonization model is sufficient for linking the notions of regularity and quasi- $r$-regularity. 
Proposition 4. Let $\mathrm{X} \subset \mathbb{Z}^{2}$ be a simply connected set. If $\mathrm{X}$ is regular, then $P_{\diamond}(\mathrm{X}) \subset \mathbb{R}^{2}$ is quasi-1-regular with margin $\sqrt{2}-1$.

Proof. We show the non-vacuity and connectedness of $P_{\diamond}(\mathrm{X}) \ominus B_{1}$ and the fact that $P_{\diamond}(\mathrm{X}) \subseteq P_{\diamond}(\mathrm{X}) \ominus B_{1} \oplus B_{\sqrt{2}}$; the same reasoning holds for $\overline{P_{\diamond}(\mathrm{X})}$. We have $B_{1} \subset C$. Then, it leads to $S^{\prime} \subset G \subseteq G \oplus C \ominus B_{1}=P_{\diamond}(\mathrm{X}) \ominus B_{1}$. The non-vacuity of $P_{\diamond}(\mathrm{X}) \ominus B_{1}$ derives from that of $S^{\prime}$ and $S$. From the definition of regularity, it is plain that $G$ is a connected subset of $\mathbb{R}^{2}$. Since $C$ is convex, $G \oplus C$ is also a connected subset of $\mathbb{R}^{2}$. But as $B_{1}$ is convex and $B_{1} \subset C, G \oplus C \ominus B_{1}=P_{\diamond}(\mathrm{X}) \ominus B_{1}$ is also connected in $\mathbb{R}^{2}$. We have $C \subset B_{\sqrt{2}}$, then $G \oplus C \subseteq G \oplus B_{\sqrt{2}}$. But we also have $G \subseteq P_{\diamond}(\mathrm{X}) \ominus B_{1}$, then $G \oplus B_{\sqrt{2}} \subseteq P_{\diamond}(\mathrm{X}) \ominus B_{1} \oplus B_{\sqrt{2}}$. It follows that $P_{\diamond}(\mathrm{X})=G \oplus C \subseteq P_{\diamond}(\mathrm{X}) \ominus B_{1} \oplus B_{\sqrt{2}}$.

\subsection{D Case}

Similarly to the case of the cubic polyhedrization, we observe that the standard marching cubes (MC) method [12], namely the 3D version of the marching squares, also fails to generate a quasi- $r$-regular polyhedron from a regular set.

Property 5. Let $\mathrm{X} \subset \mathbb{Z}^{3}$ be a simply connected, regular set. Let $P_{\diamond}(\mathrm{X})$ be the polyhedron generated from $\mathrm{X}$ by MC polyhedrization. Then $P_{\diamond}(\mathrm{X})$ is never quasi-1-regular with $\operatorname{margin} \frac{2}{\sqrt{3}}-1$.

To prove this property, it is sufficient to observe that there exists a vertex $v$ on a convex part of $P_{\diamond}(\mathrm{X})$ (such vertex exists, as $\mathrm{X}$ is finite and $P_{\diamond}(\mathrm{X})$ is then bounded), such that the distance between $v$ and $P_{\diamond}(\mathrm{X}) \ominus B_{1}$ is $\frac{\sqrt{6}}{2}>\frac{2}{\sqrt{3}}$. Thus, $v$ does not belong to $P_{\diamond}(\mathrm{X}) \ominus$ $B_{1} \oplus B_{\frac{2}{\sqrt{3}}}$.

\section{Conclusion}

In this article, we observed that the notion of quasi-r-regularity allows one to define polygons / polyhedra that preserve their topology under digitization in $\mathbb{Z}^{n}$ for $n=2,3$ (this property is no longer true in $\mathbb{Z}^{n}, n \geq 4$ ). As a consequence, building a quasi$r$-regular polygon / polyhedron from a digital set in $\mathbb{Z}^{2}$ or $\mathbb{Z}^{3}$ for handling topologypreserving rigid motions is relevant. In this context, we established that two simple polygonization models (cubic model and marching squares) can link the notions of regularity and quasi-r-regularity in $\mathbb{Z}^{2}$. However, in $3 \mathrm{D}$, the corresponding models fail to generate quasi-r-regular polyhedra in $\mathbb{R}^{3}$. Our further works will consist of investigating other kinds of polyhedrization devoted to 3D regular images.

\section{References}

1. Bazin, P.L., Ellingsen, L.M., Pham, D.L.: Digital homeomorphisms in deformable registration. In: Karssemeijer, N., Lelieveldt, B. (eds.) Information Processing in Medical Imaging. pp. 211-222. Springer Berlin Heidelberg (2007)

2. Bertrand, G.: On P-simple points. Comptes Rendus de l'Académie des Sciences, Série Mathématique I(321), 1077-1084 (1995) 
3. Bertrand, G., Malandain, G.: A new characterization of three-dimensional simple points. Pattern Recognition Letters 15(2), 169-175 (1994)

4. Boutry, N., Géraud, T., Najman, L.: A tutorial on well-composedness. Journal of Mathematical Imaging and Vision 60(3), 443-478 (2018)

5. Khalimsky, E.: Topological structures in computer science. Journal of Applied Mathematics and Simulation 1(1), 25-40 (1987)

6. Kong, T.Y.: A digital fundamental group. Computers \& Graphics 13(2), 159-166 (1989)

7. Kong, T.Y., Rosenfeld, A.: Digital topology: Introduction and survey. Computer Vision, Graphics, and Image Processing 48(3), 357-393 (1989)

8. Kovalevsky, V.A.: Finite topology as applied to image analysis. Computer Vision, Graphics, and Image Processing 46(2), 141-161 (1989)

9. Largeteau-Skapin, G., Andres, E.: Discrete-Euclidean operations. Discrete Applied Mathematics 157(3), 510-523 (2009)

10. Latecki, L.J.: 3D well-composed pictures. Graphical Models and Image Processing 59(3), 164-172 (1997)

11. Latecki, L.J., Eckhardt, U., Rosenfeld, A.: Well-composed sets. Computer Vision and Image Understanding 5(1), 70-83 (1995)

12. Lorensen, W.E., Cline, H.E.: Marching cubes: A high-resolution 3D surface construction algorithm. ACM SIGGRAPH Computer Graphics 21(4), 163-169 (1987)

13. Maunder, C.R.F.: Algebraic Topology. Dover (1996)

14. Mazo, L., Passat, N., Couprie, M., Ronse, C.: Digital imaging: A unified topological framework. Journal of Mathematical Imaging and Vision 44(1), 19-37 (2012)

15. Mazo, L., Passat, N., Couprie, M., Ronse, C.: Paths, homotopy and reduction in digital images. Acta Applicandae Mathematicae 113(2), 167-193 (2011)

16. Ngo, P., Passat, N., Kenmochi, Y., Debled-Rennesson, I.: Geometric preservation of 2D digital objects under rigid motions. Journal of Mathematical Imaging and Vision https://doi.org/10.1007/s10851-018-0842-9

17. Ngo, P., Passat, N., Kenmochi, Y., Debled-Rennesson, I.: Convexity invariance of voxel objects under rigid motions. In: International Conference on Pattern Recognition. pp. 11571162 (2018)

18. Ngo, P., Passat, N., Kenmochi, Y., Talbot, H.: Topology-preserving rigid transformation of 2D digital images. IEEE Transactions on Image Processing 23(2), 885-897 (2014)

19. Passat, N., Mazo, L.: An introduction to simple sets. Pattern Recognition Letters 30(15), 1366-1377 (2009)

20. Pavlidis, T.: Algorithms for Graphics and Image Processing. Berlin: Springer, and Rockville: Computer Science Press (1982)

21. Rosenfeld, A.: Adjacency in digital pictures. Information and Control 26(1), 24-33 (1974)

22. Rosenfeld, A.: Digital topology. The American Mathematical Monthly 86(8), 621-630 (1979)

23. Rosenfeld, A., Kong, T.Y., Nakamura, A.: Topology-preserving deformations of two-valued digital pictures. Graphical Models and Image Processing 60(1), 24-34 (1998)

24. Stelldinger, P., Latecki, L.J., Siqueira, M.: Topological equivalence between a 3D object and the reconstruction of its digital image. IEEE Transactions on Pattern Analysis and Machine Intelligence 29(1), 126-140 (2007)

25. Stelldinger, P., Terzic, K.: Digitization of non-regular shapes in arbitrary dimensions. Image and Vision Computing 26(10), 1338-1346 (2008)

26. Zitová, B., Flusser, J.: Image registration methods: A survey. Image and Vision Computing 21(11), 977-1000 (2003) 